\title{
Congenital Cystic Lesions of the Biliary Tree
}

\section{Inês Santiago ${ }^{1}$ \\ Rui Loureiro ${ }^{2}$ \\ Luis Curvo-Semedo ${ }^{3}$ \\ Cristina Marques ${ }^{3}$ \\ Francisco Tardáguila ${ }^{4}$ \\ Celso Matos ${ }^{5}$ \\ Filipe Caseiro-Alves ${ }^{3}$}

OBJECTIVE. The purpose of this essay is to illustrate the imaging findings of congenital cystic lesions of the biliary tract.

CONCLUSION. Congenital cystic lesions of the biliary tract include ductal plate malformations and choledochal cysts and can be recognized with characteristic imaging findings and basic knowledge of the embryologic development of the biliary tree.

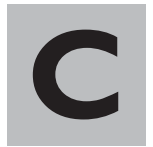
ongenital cystic lesions of the biliary tract affect the intrahepatic and extrahepatic bile ducts. Cystic diseases affecting the intrahepatic bile ducts result from ductal plate malformations and include Von Meyenburg complexes, congenital hepatic fibrosis, polycystic liver disease, and Caroli disease. Congenital cystic lesions of the extrahepatic bile ducts correspond to choledochal cysts, which may be secondary to abnormalities of the pancreaticobiliary junction. Most congenital cystic diseases of the biliary tract are known predisposing factors for the development of recurrent pancreatitis, cholangitis, lithiasis, and malignancy and should not go unrecognized. The objective of this essay is to illustrate congenital cystic lesions of the biliary tract on the basis of knowledge of embryologic development.

\section{Embryology}

During the fourth embryonic week, the hepatic diverticulum, an endodermal outgrowth of the primitive midgut, arises $[1,2]$. The cranial part of the hepatic diverticulum (pars hepatica) develops into the liver primordium and common hepatic duct. The caudal part divides into a superior bud (pars cystica), from which the gallbladder and cystic duct arise, and an inferior bud, the origination of the ventral bud of the pancreas [1]. During the sixth embryonic week, the choledochus and ventral bud of the pancreas rotate $180^{\circ}$ clockwise around the duodenum and assume their definitive positions [1] (Fig. 1). The liver primordium generates liver precursor cells. By the eighth gestational week, some of these cells differentiate into epithelial cells arranged as double-layer cylindric structures in the mesenchyme adjacent to the portal vein branches called the ductal plates [2]. By the 12th gestational week, the ductal plates have started remodeling, and by week 20 immature bile ducts and peribiliary glands are seen at the liver hilum. These glands are mature by the end of gestation or the early postnatal period [2] (Fig. 2).

\section{Congenital Cystic Lesions of the Intrahepatic Bile Ducts}

If remodeling of the ductal plates is abnormal, malformations occur. These malformations are due to abnormal persistence of embryonic bile ducts and may present as cystic noncommunicating lesions or supernumerary ectatic bile ducts (Fig. 3). The caliber of malformed ducts determines the type of ductal plate malformation. If small interlobular bile ducts are affected, Von Meyenburg complexes or congenital hepatic fibrosis results. If medium-sized intrahepatic ducts are affected, autosomal dominant polycystic liver disease results. Caroli disease is the result of ductal plate malformation of large intrahepatic bile ducts [3].

Von Meyenburg complexes, or biliary hamartomas, result from failure of involution of embryonic bile ducts and have a reported incidence as high as $5.6 \%$. Lesions are usually scattered, multiple, and smaller than $10 \mathrm{~mm}$ [4] and can be solid, cystic, or mixed [5]. They do not communicate with the biliary tree. At ultrasound, the lesions usually present as tiny hyperechoic, hypoechoic, or mixed lesions with comet-tail echoes [4]. At CT, the lesions 


\section{Santiago et al.}

are small and hypoattenuating. At MRI, the lesions are hypointense on T1-weighted images and hyperintense on T2-weighted images [3]. MRCP may show the lesions more clearly in terms of number and shape [4]. Biliary hamartomas typically do not enhance after IV contrast administration [3, 4] (Fig. 4). Uncommonly, homogeneous enhancement [4] or a peripheral enhancing rim may be seen [6]. The differential diagnosis includes simple hepatic cysts and metastatic lesions, which are rarely uniform in size, attenuation, or signal intensity [3].

Congenital hepatic fibrosis may present in isolation but is usually associated with autosomal recessive polycystic kidney disease [7]. It is characterized by enlargement of portal spaces due to the presence of fibrosis and numerous, more or less ectatic, abnormal bile ducts communicating with the biliary tree [8]. Bile duct ectasia is not macroscopically visible unless Caroli disease is present [8]. The main feature of the disease is portal hypertension without liver insufficiency, but progression of fibrosis can evolve to true cirrhosis [9]. Morphologic changes in the liver, namely, hypertrophy of the lateral segments of the left lobe and normal volume or hypertrophy of segments IVa and IVb, associated ductal plate malformations, varices, splenomegaly, and renal abnormalities, are frequently found in combination in patients with congenital hepatic fibrosis [9].

Autosomal dominant polycystic liver disease can occur in isolation or be associated with autosomal dominant polycystic kidney disease, which occurs in approximately $50 \%$ of cases [10]. Liver cysts originate from biliary microhamartomas lined with functional biliary epithelium and do not communicate with the biliary tree. They usually become apparent after puberty and grow in size and number at variable rates with age [11, 12]. Women are usually more severely affected $[12,13]$. The presence of more than 20 liver cysts (four, if there is a family history of cysts) is diagnostic [13] (Fig. 5). Most patients have no symptoms. Imaging studies usually show multiple liver cysts of various sizes, as large as $12 \mathrm{~cm}$, in an enlarged liver $[3,12]$. Ultrasound images show cysts as anechoic lesions with well-defined thin walls. CT scans show homogeneous, water-attenuation, nonenhancing lesions. MRI shows T1hypointense, T2-hyperintense lesions. The presence of echogenic material in a cyst at ultrasound, spontaneous high attenuation at $\mathrm{CT}$, and internal changes in signal intensity at MRI should point to cyst complications, namely, hemorrhage and infection. Complications can also cause calcification of cyst walls. Associated dilatation of the intrahepatic and extrahepatic bile ducts may also be found in autosomal dominant polycystic liver disease [3, 12].

Caroli disease is thought to be caused by incomplete ductal plate remodeling that results in abnormal persistence of ductal plate remnants [14]. It is a rare autosomal recessive disease characterized by segmental or, less often, diffuse communicating nonobstructive saccular or fusiform dilatation of large intrahepatic bile ducts (up to $5 \mathrm{~cm}$ ), often containing calculi, sludge, or both [3, 15]. Cholangitis, cirrhosis, and cholangiocarcinoma are known complications [3, 15]. The central dot sign is typical and represents cystic enveloping of portal tract vessels [3] (Fig. 6). Caroli disease can be difficult to differentiate from polycystic liver disease and obstructive bile duct dilatation, in which case MRCP may be particularly helpful. Liverspecific hepatobiliary MRI contrast agents can also be used to prove communication with the biliary tree.

Caroli syndrome is said to be present when Caroli disease and congenital hepatic fibrosis coexist (Figs. 7 and 8) and is more common than Caroli disease alone [16]. The two conditions may represent different stages of the same disease [16].

\section{Congenital Cystic Lesions of the Extrahepatic Bile Ducts}

Choledochal cysts are rare abnormalities manifested by cystic dilatation of the extrahepatic and/or intrahepatic biliary tree. The incidence is higher in Asia (1/1000) than in western countries $(1 / 10,0000-150,000)$ [17]. Women are more commonly affected [18, 19]. Choledochal cysts are classified according to the Todani modification of the Alonso-Lej classification (Fig. 9), which some authors have challenged, claiming that different subtypes of choledochal cysts (Figs. 10-14) have different causes and carcinogenicity [17]. Types I and IVA, for instance, may represent variations of the same disease because all type I cysts have some degree of intrahepatic bile duct dilatation [17].

The clinical presentation of choledochal cysts occurs before the age of 10 years in $80 \%$ of patients, usually the result of complications, namely cholangitis and pancreatitis [20]. Another known complication is malignancy $[20,21]$. The overall risk of cancer in choledochal cysts is $10-15 \%$, the extrahepatic bile ducts and gallbladder being the most common sites [21]. Although the cause is unclear, it is widely accepted that choledochal cysts arise from an anomalous pancreaticobiliary ductal junction, particularly a long common channel, which is seen in $50-80 \%$ of patients $[17,19,22]$. Anomalous pancreaticobiliary ductal junction may allow mixture of pancreatic and biliary juices, which activates pancreatic enzymes leading to consequent inflammation and weakening of the duct wall [17, 22]. In $22 \%$ of cases, anomalous pancreaticobiliary ductal junction is seen without dilatation, but patients are still at high risk of carcinogenesis, particularly of gallbladder cancer [23].

\section{Conclusion}

Congenital cystic lesions of the intrahepatic bile ducts are the result of ductal plate malformations. The clinical significance is varied, ranging from none in biliary hamartomas to high risk of cholangiocarcinoma in Caroli disease. Congenital cystic lesions of the extrahepatic bile ducts (choledochal cysts) are predisposing factors for cholangitis, pancreatitis, and malignancy. Although classically grouped together, these lesions can represent different abnormalities with different causes and carcinogenicities. Basic knowledge of the embryologic development of the biliary tree is essential for recognizing and understanding the imaging findings of these two groups of disorders.

\section{References}

1. Ando H. Embryology of the biliary tract. Dig Surg 2010; 27:87-89

2. Terada T, Kitamura Y, Nakanuma Y. Normal and abnormal development of the human intrahepatic biliary system: a review. Tohoku J Exp Med 1997; 181:19-32

3. Brancatelli G, Federle MP, Vilgrain V, Vullierme MP, Marin D, Lagalla R. Fibropolycystic liver disease: CT and MR imaging findings. RadioGraphics 2005; 25:659-670

4. Zheng RQ, Zhang B, Kudo M, Onda H, Inoue T. Imaging findings of biliary hamartomas. World $J$ Gastroenterol 2005; 28;11:6354-6359

5. Cheung YC, Tan CF, Wan YL, Lui KW, Tsai CC. MRI of multiple biliary hamartomas. Br J Radiol 1997; 70:527-529

6. Elsayes KM, Narra VR, Yin Y, Mukundan G, Lammle M, Brown JJ. Focal hepatic lesions: diagnostic value of enhancement pattern approach with contrast-enhanced 3D gradient echo MR imaging. RadioGraphics 2005; 25:1299-1320

7. Desmet VJ. Pathogenesis of ductal plate abnormalities. Mayo Clin Proc 1998; 73:80-89 


\section{Congenital Biliary Cysts}

8. Dhumeaux D. Congenital cystic diseases of the intra and extrahepatic bile ducts. Gastroenterol Clin Biol 2005; 29:878-882

9. Zeitoun D, Brancatelli G, Colombat M, et al. Congenital hepatic fibrosis: CT findings in 18 adults. Radiology 2004; 231:109-116

10. Housset C. Cystic liver diseases: genetics and cell biology. Gastroenterol Clin Biol 2005; 29:861-869

11. Morgan DE, Lockhart ME, Canon CL, Holcombe MP, Bynon JS. Polycystic liver disease: multimodality imaging for complications and transplant evaluation. RadioGraphics 2006; 26:1655-1668

12. Qian Q. Isolated polycystic liver disease. $A d v$ Chronic Kidney Dis 2010; 17:181-189

13. Drenth JP, Chrispijn M, Bergmann C. Congenital fibrocystic liver diseases. Best Pract Res Clin Gastroenterol 2010; 24:573-584
14. Desmet VJ. Cystic diseases of the liver: from embryology to malformations. Gastroenterol Clin Biol 2005; 29:858-860

15. Yonem O, Bayraktar Y. Clinical characteristics of Caroli's disease. World J Gastroenterol 2007; 13:1930-1933

16. Yonem O, Bayraktar Y. Clinical characteristics of Caroli's syndrome. World J Gastroenterol 2007; 13:1934-1937

17. Singham J, Yoshida EM, Scudamore CH. Choledochal cysts. Part 1. Classification and pathogenesis. Can J Surg 2009; 52:434-440

18. Mortelé KJ, Rocha TC, Streeter JL, Taylor AJ. Multimodality imaging of pancreatic and biliary congenital anomalies. RadioGraphics 2006; 26 : 715-731

19. Matos C, Nicaise N, Devière J, et al. Choledochal cysts: comparison of findings at MR cholangiopancreatography and endoscopic retrograde cholangiopancreatography in eight patients. Radiology 1998; 209:443-448

20. Singham J, Yoshida EM, Scudamore CH. Choledochal cysts. Part 2. Diagnosis. Can J Surg 2009; 52:506-511

21. Singham J, Yoshida EM, Scudamore CH. Choledochal cysts. Part 3. Management. Can J Surg 2010; 53:51-56

22. Tan SS, Tan NC, Ibrahim S, Tay KH. Management of adult choledochal cyst. Singapore Med J 2007; 48:524-527

23. Funabiki T, Matsubara T, Miyakawa S, Ishihara S. Pancreaticobiliary maljunction and carcinogenesis to biliary and pancreatic malignancy. Langenbecks Arch Surg 2009; 394:159-169

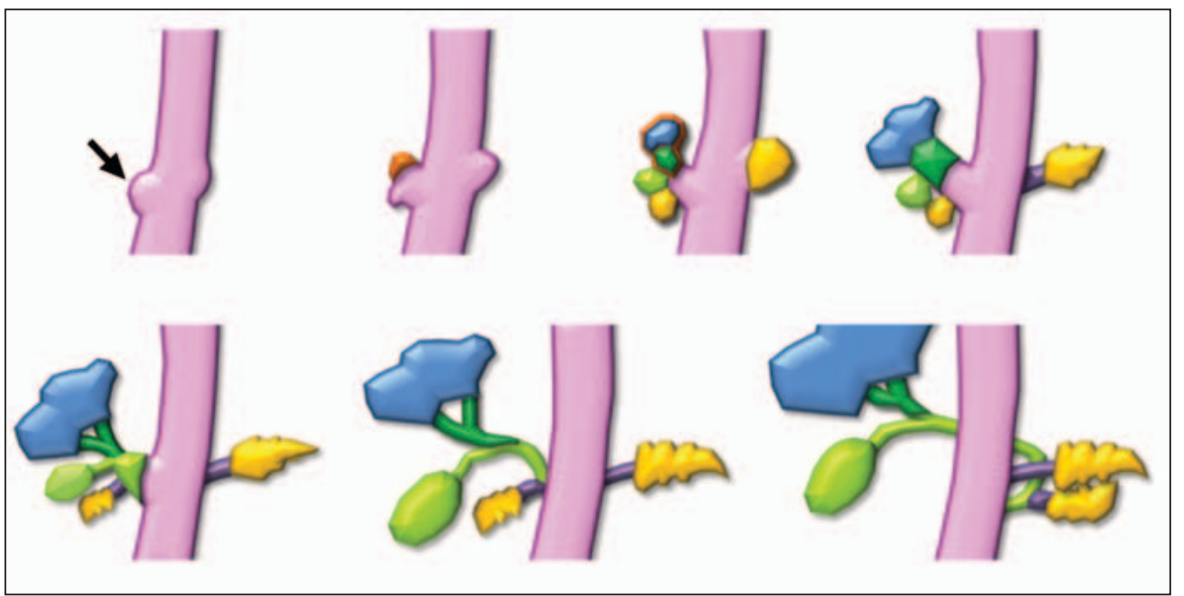

Fig. 1-Schematic shows development of biliary tract. At beginning of fourth gestational week, hepatic diverticulum (arrow) arises from ventral wall of primitive midgut. Pars hepatica (orange) originates from cranial part of hepatic diverticulum and develops into liver primordium (b/ue) and common hepatic duct (dark green). Caudal part of pars hepatica divides into superior bud (light green) and inferior bud (yellow). Gallbladder, cystic duct, and choledochus originate from superior bud (pars cystica). Inferior bud develops into ventral bud of pancreas. During sixth gestational week, choledochus and ventral bud of pancreas rotate $180^{\circ}$ clockwise around duodenum and come to lie in their definitive positions.

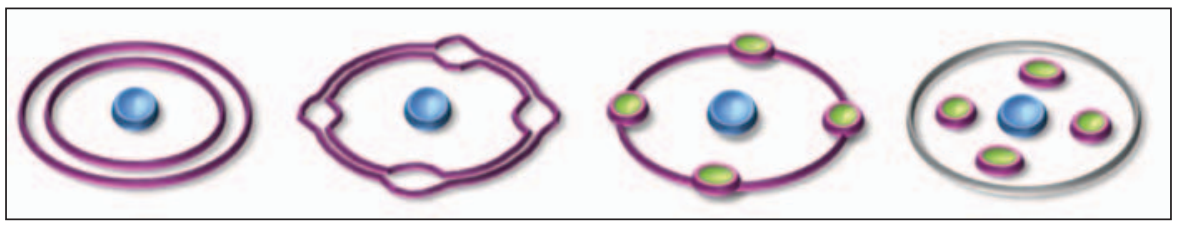

Fig. 2-Schematic shows development of biliary tract. During eighth gestational week, ductal plate (purple), which consists of double layer of flattened cuboidal epithelial cells, becomes apparent in mesenchyme surrounding portal vein branches (b/ue). By 12th gestational week, plate starts to remodel. Parts of two layers of cuboidal epithelial cells fuse and are reabsorbed. Unfused portions constitute definitive bile ducts, which are incorporated in portal mesenchyme [14].

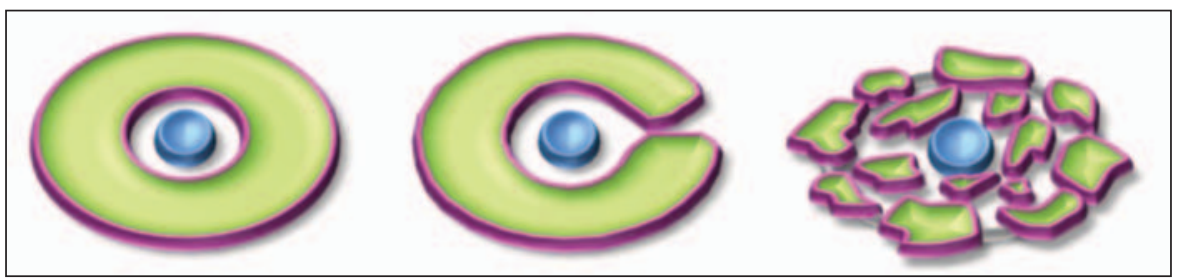

Fig. 3-Schematic shows ductal plate malformations. Anomalies consist of complete lack of remodeling of ductal plate, in which continuous dilated duct encircles portal vein radicle (/eft), or incomplete remodeling of ductal plate, which results in either single dilated bile duct with polypoid projection of portal mesenchyme (center) or interrupted circle of ectatic bile ducts (right) [14]. 


\section{Santiago et al.}
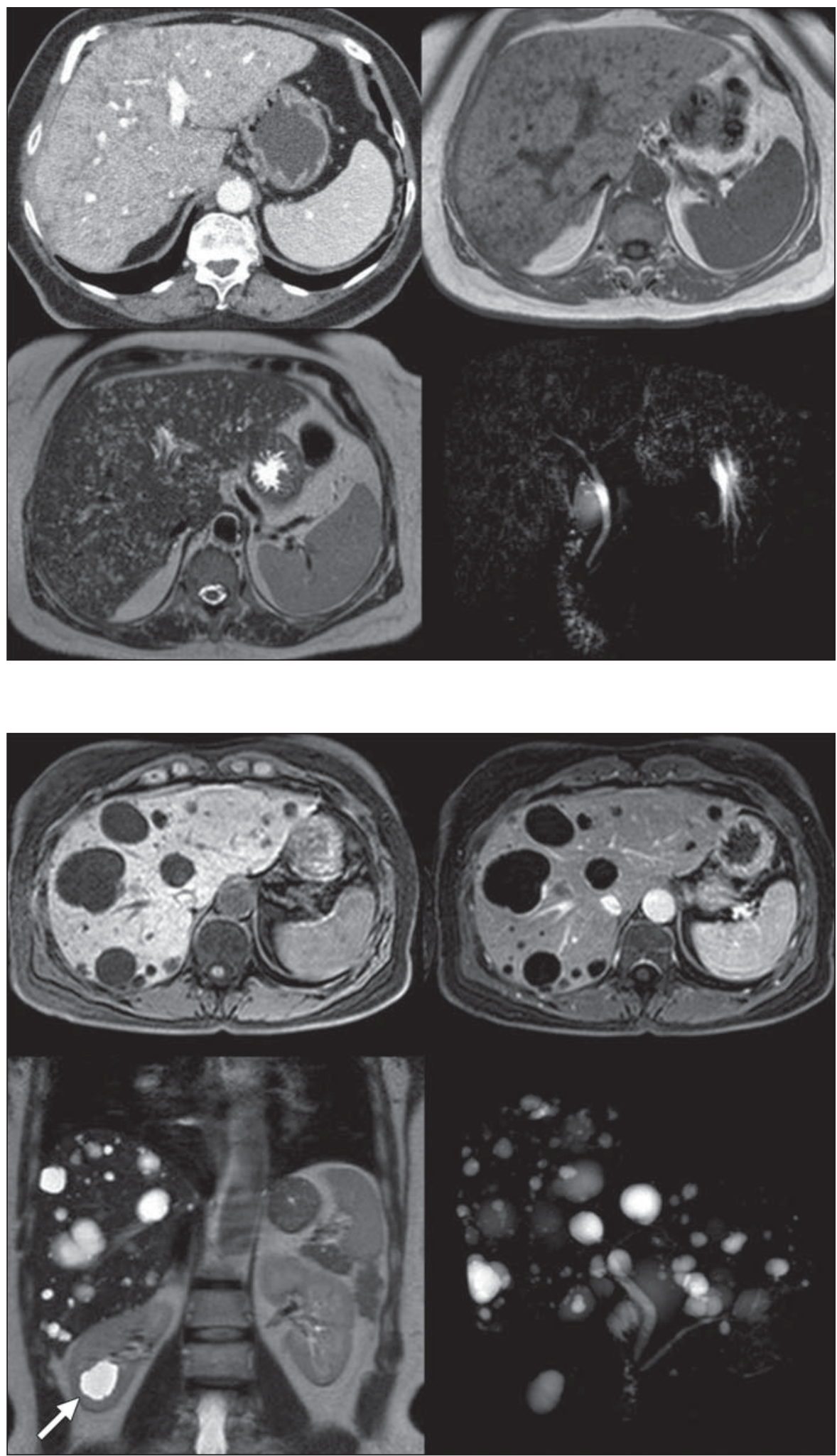

Fig. 4-Von Meyenburg complexes. 53-year-old woman with abdominal pain due to gastroenteritis. Abdominal ultrasound images showed scattered hyperechoic and hypoechoic submillimetric lesions in liver. Abdominal CT image (top left) shows multiple subcentimeter hypodense nonenhancing lesions. T1-weighted MR image (top right) shows lesions as hypointense foci, and T2-weighted image (bottom left), as hyperintense foci. Thick-section singleshot fast spin-echo heavily T2-weighted MR image (bottom right) shows no communication with biliary tree.
Fig. 5-49-year-old man with known autosoma dominant polycystic liver disease undergoing routine evaluation. T1-weighted MR image (top left) shows more than 20 hepatic cysts of various sizes as hypointense lesions. MR image obtained after IV gadolinium administration (top right) shows no lesion enhancement. T2-weighted image (bottom left) shows hyperintense lesions. Thick-section single-shot fast spin-echo heavily T2-weighted MR image (bottom right) shows no communication with biliary tree. Only one renal cyst (arrow) was found on right kidney. There was therefore no evidence of autosomal dominant polycystic kidney disease. 


\section{Congenital Biliary Cysts}
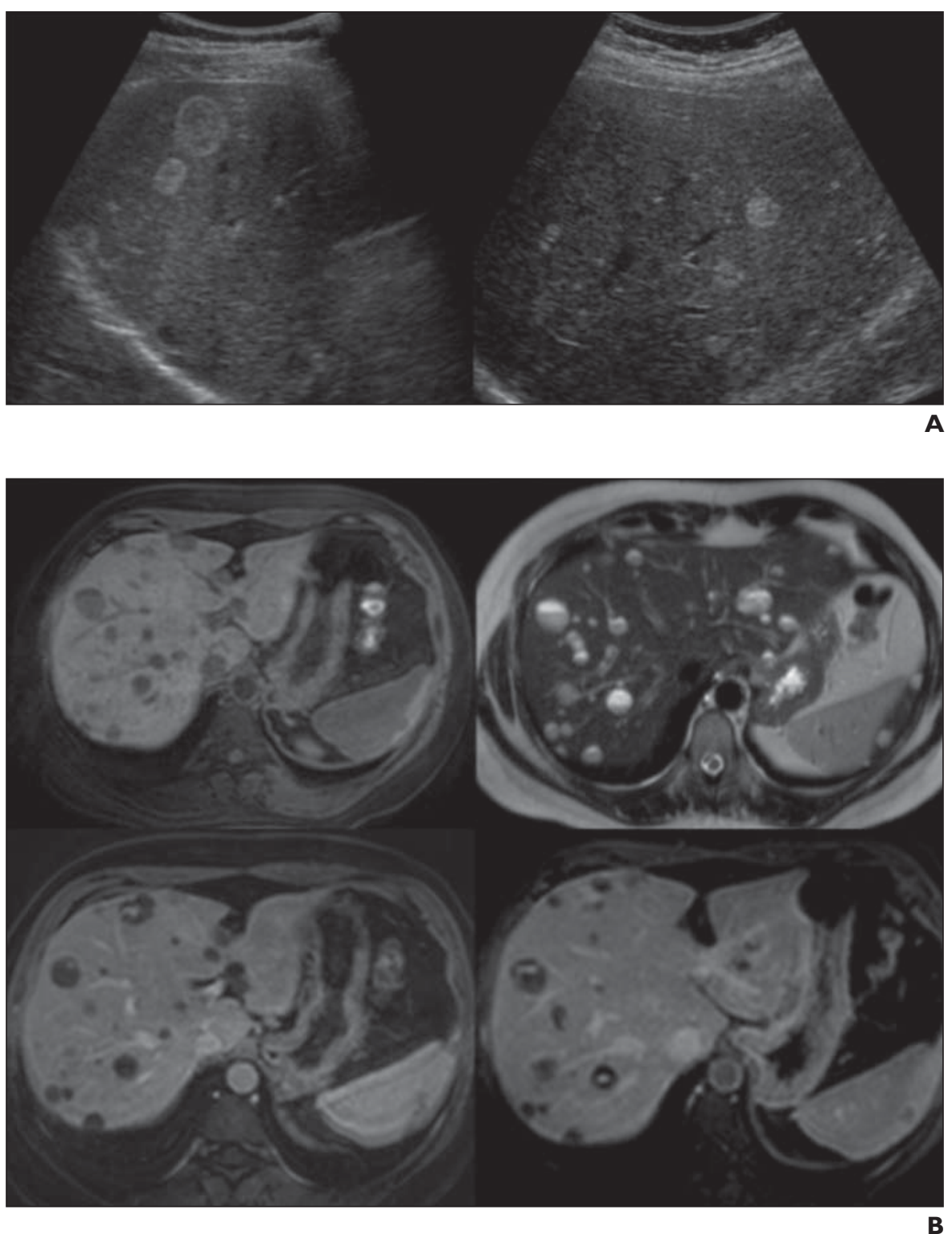

Fig. 6-Caroli disease. 45-year-old man with abnormal liver function test results.

A, Abdominal ultrasound images show multiple round heterogeneously hyperechoic well-defined liver lesions of various sizes, simulating hemangiomas or metastatic lesions.

B, T1-weighted MR image (top left) shows hypointense and T2-weighted image (top right) shows hyperintense lesions. Most lesions had fluid-fluid levels, which disproved cystic nature. Portal venous phase images after gadoxetate disodium administration (bottom) show multiple central dot signs.

(Fig. 6 continues on next page) 


\section{Santiago et al.}

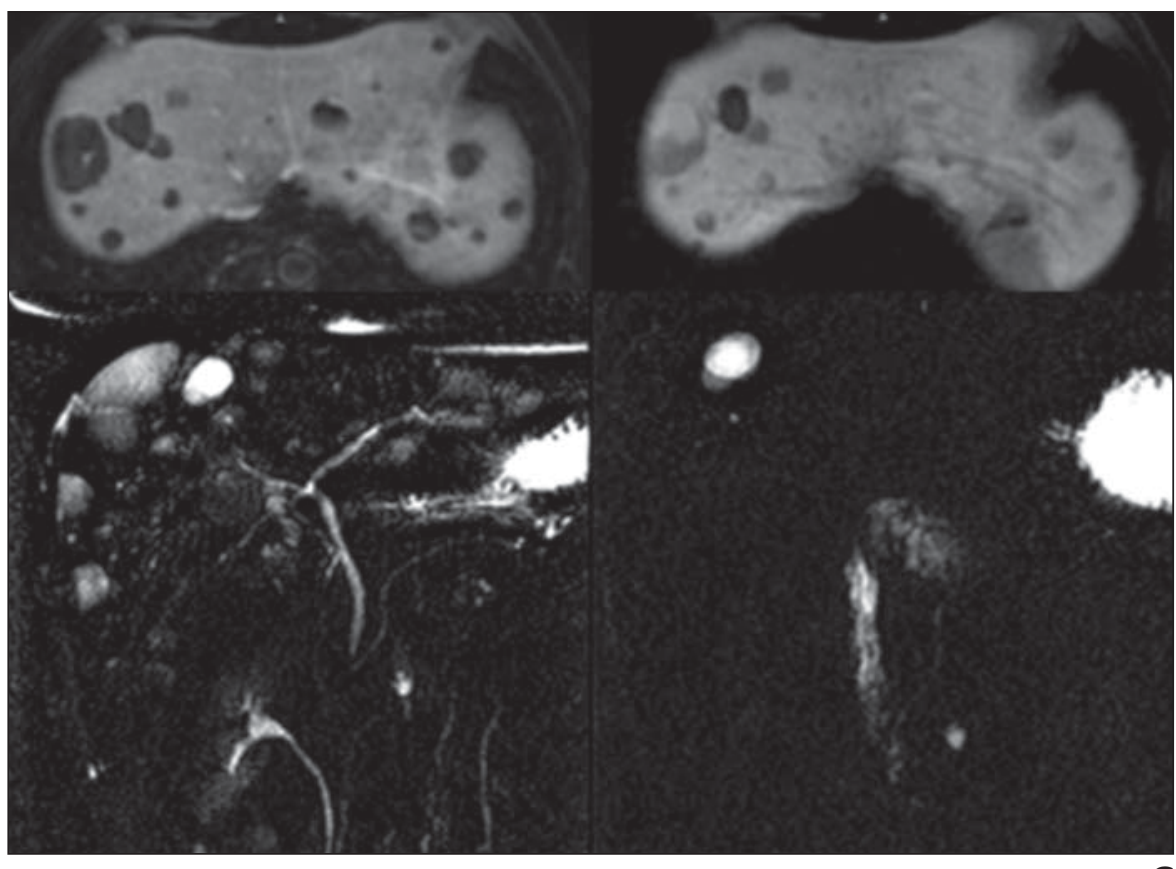

C

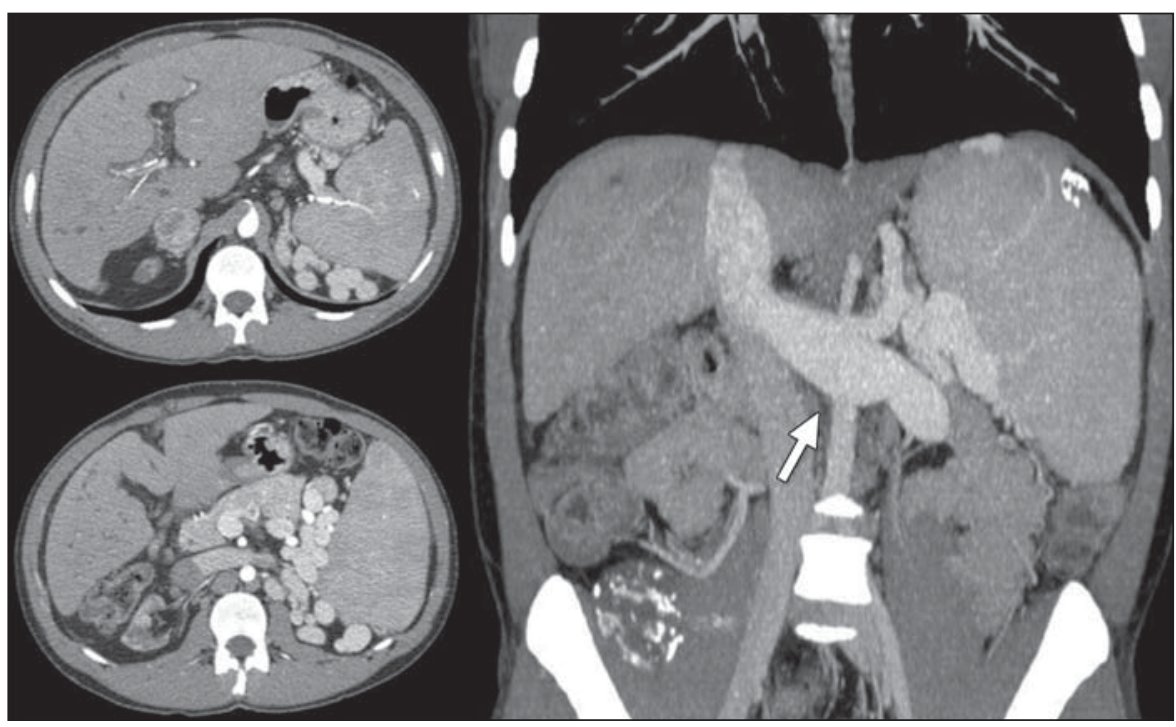

A
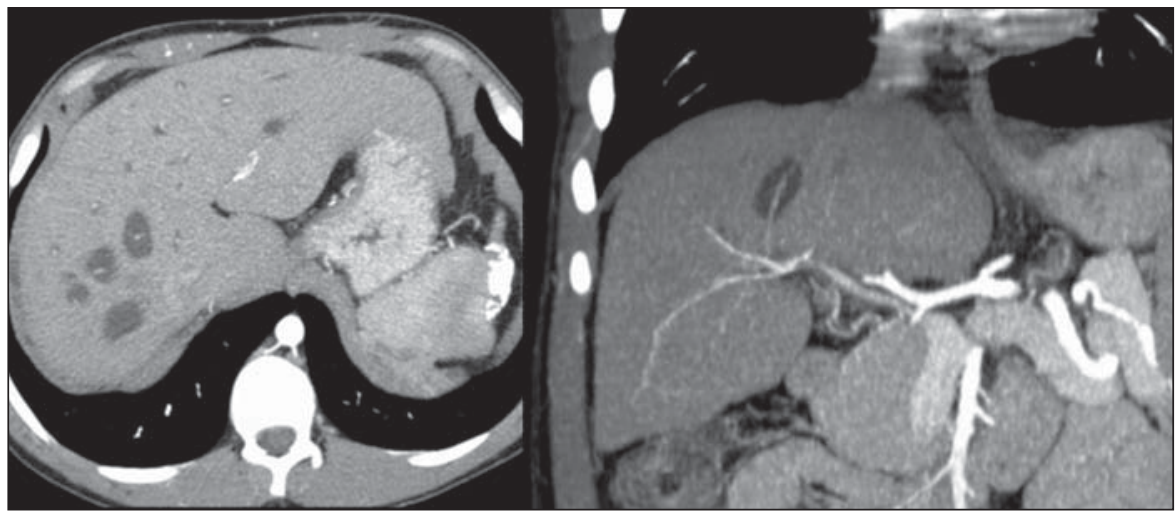

Fig. 6 (continued)—Caroli disease. 45-year-old man with abnormal liver function test results.

C. Hepatobiliary phase MR images (top) show most lesions have partially filled with contrast material, proving communication with biliary tree. Single-shot fast spin-echo heavily T2-weighted MR images obtained before (bottom left) and after (bottom right) gadoxetate disodium administration (hepatobiliary phase) also show communication with biliary tree. T2-shortening effect of excreted contrast agent nulls signal intensity of bile in both ducts and communicating lesions. Single liver cyst remains visible, possibly corresponding to biliary cyst.
Fig. 7-Caroli syndrome. 25-year-old man with known congenital hepatic fibrosis undergoing evaluation before liver transplant.

A. Contrast-enhanced portal venous phase, axial (left) and coronal maximum intensity projection (MIP) reformatted (right) CT images show morphologic changes in liver: right lobe atrophy and left lobe hypertrophy, particularly of lateral segments. Multiple tortuous varices (/eft) drain to systemic circulation through splenorenal shunts, resulting in marked ectasia of left renal vein (arrow). Splenomegaly also is apparent (right).

B, Axial (left) and coronal MIP reformatted (right) CT images show coexistent Caroli disease as multiple cystic lesions enveloping portal vein branches (central dot sign). 


\section{Congenital Biliary Cysts}

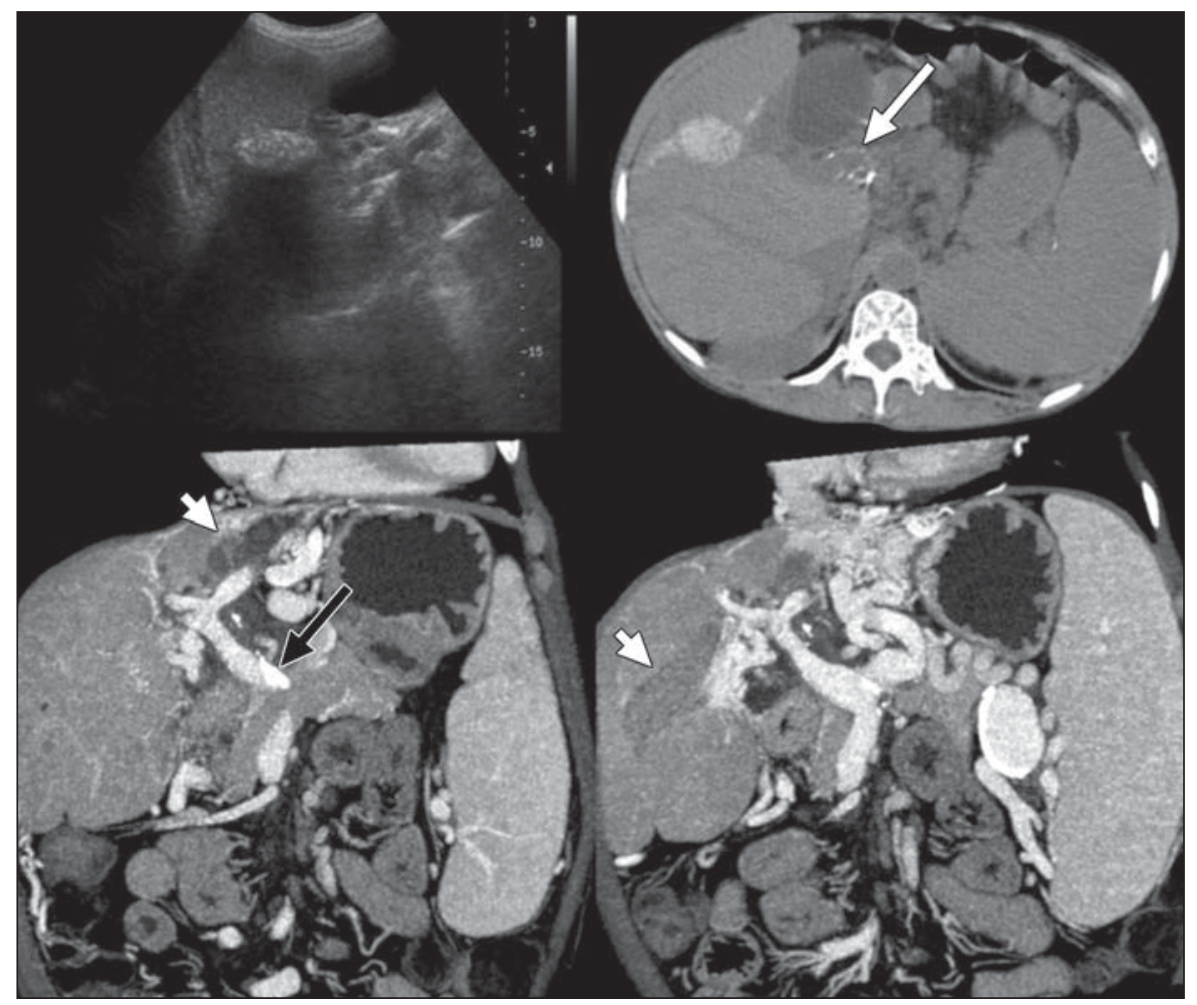

Fig. 8-Caroli syndrome. 48-year-old man with clinical signs of portal hypertension. Abdominal ultrasound image (top left) shows oval hyperechoic heterogeneous structure with posterior acoustic shadowing in right liver lobe corresponding to markedly dilated intrahepatic bile duct filled with calculi. Unenhanced CT image (top right) shows high-attenuation calculi (arrow). Contrastenhanced portal venous phase coronal multiplanar reformatted images (bottom) depict marked dilatation of intrahepatic bile ducts in both lobes. Associated findings of portal hypertension, namely, splenomegaly and tortuous perigastric and perisplenic venous collaterals, are evident, as are signs of chronic portal vein thrombosis, namely, peripheral partially calcified thrombus (arrows).

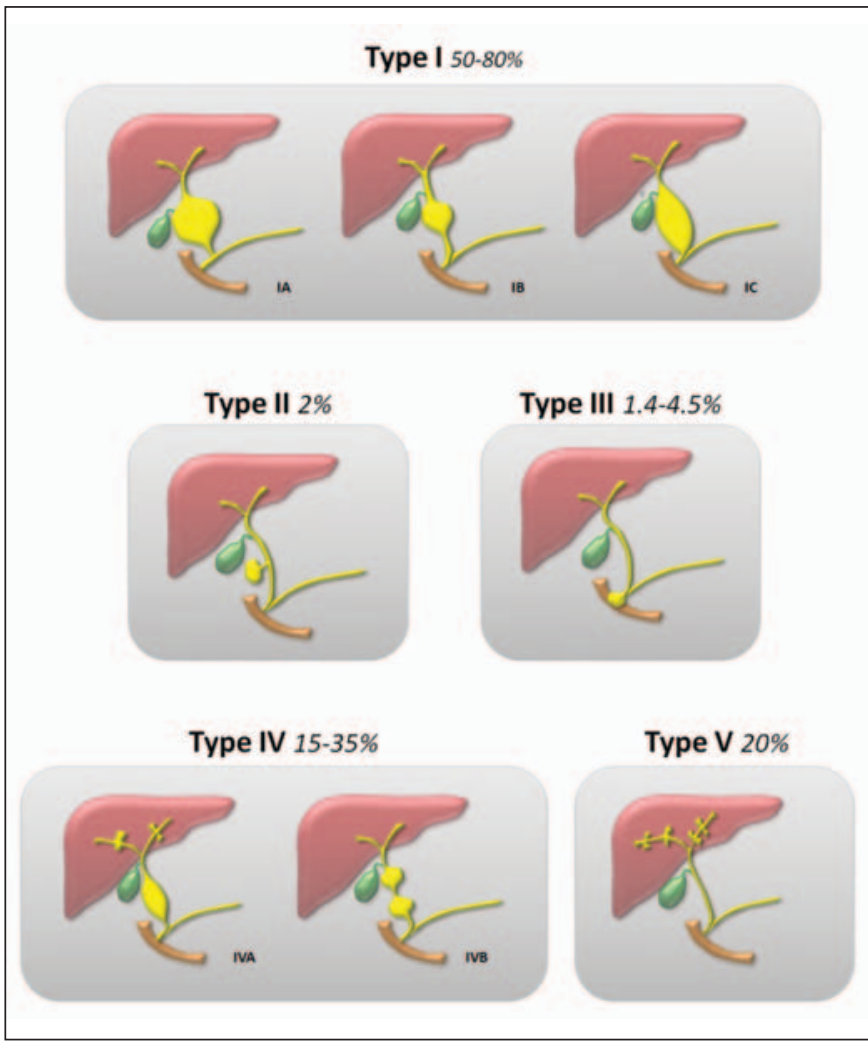

Fig. 9-Schematics show Todani modification of Alonso-Lej classification and relative percentage of occurrence of each type of choledochal cyst. Type IA is marked cystic dilatation of entire extrahepatic bile duct; IB, focal segmental dilatation of extrahepatic bile duct, which can occur at any level but is usually distal to cystic duct insertion; IC, smooth fusiform dilatation of entire extrahepatic bile duct; II, discrete diverticulum of extrahepatic bile duct; III, dilatation of distal common bile duct confined to wall of duodenum, often bulging into duodenal lumen (choledochocele); IVA, multiple sites of dilatation of both extrahepatic and intrahepatic biliary tree; IVB, multiple sites of dilatation of extrahepatic bile duct only (string-of-beads appearance); V, multiple sites of saccular or cystic dilatation of only intrahepatic biliary tree (Caroli disease or communicating cavernous ectasia) [17]. 


\section{Santiago et al.}

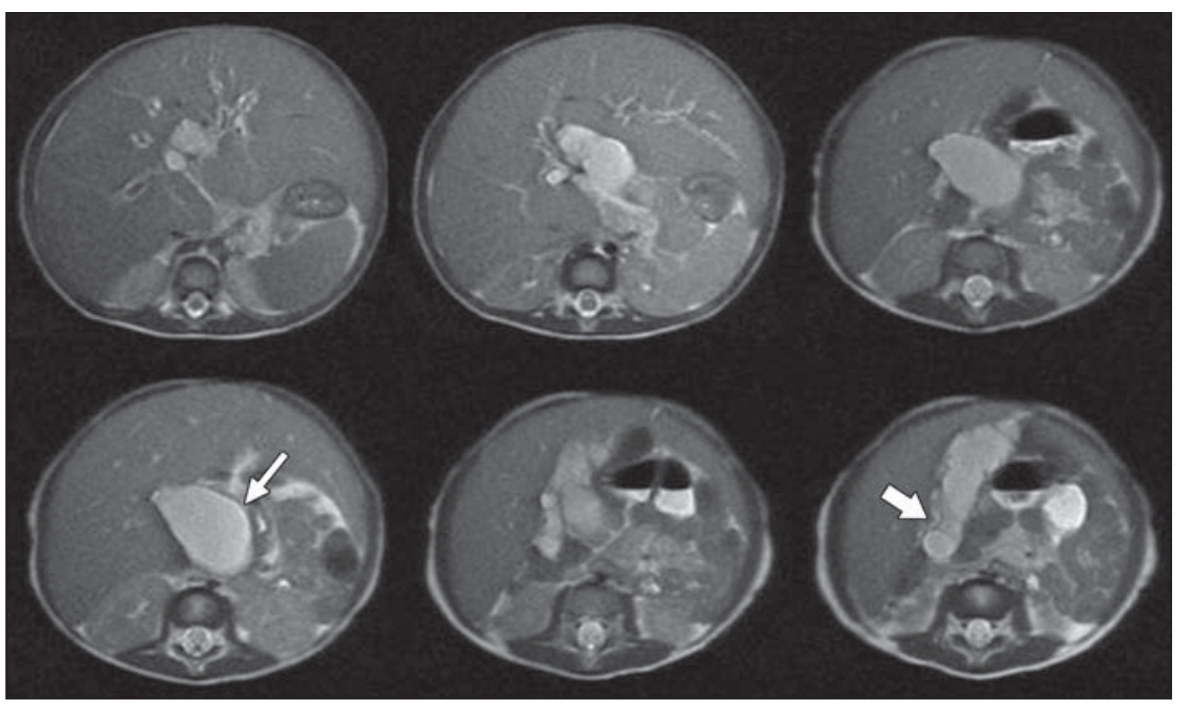

Fig. 10-Type IC choledochal cyst. 4-year-old girl with jaundice and abdominal pain.

A, Axial T2-weighted HASTE MR images show marked tortuous fusiform dilatation of extrahepatic bile duct (arrows).

B, Coronal T2-weighted HASTE (top left), steadystate free precession (top right), and thick-section single-shot fast spin-echo heavily T2-weighted (bottom) MR images show cystic duct draining to dilated portion of main bile duct (arrow).

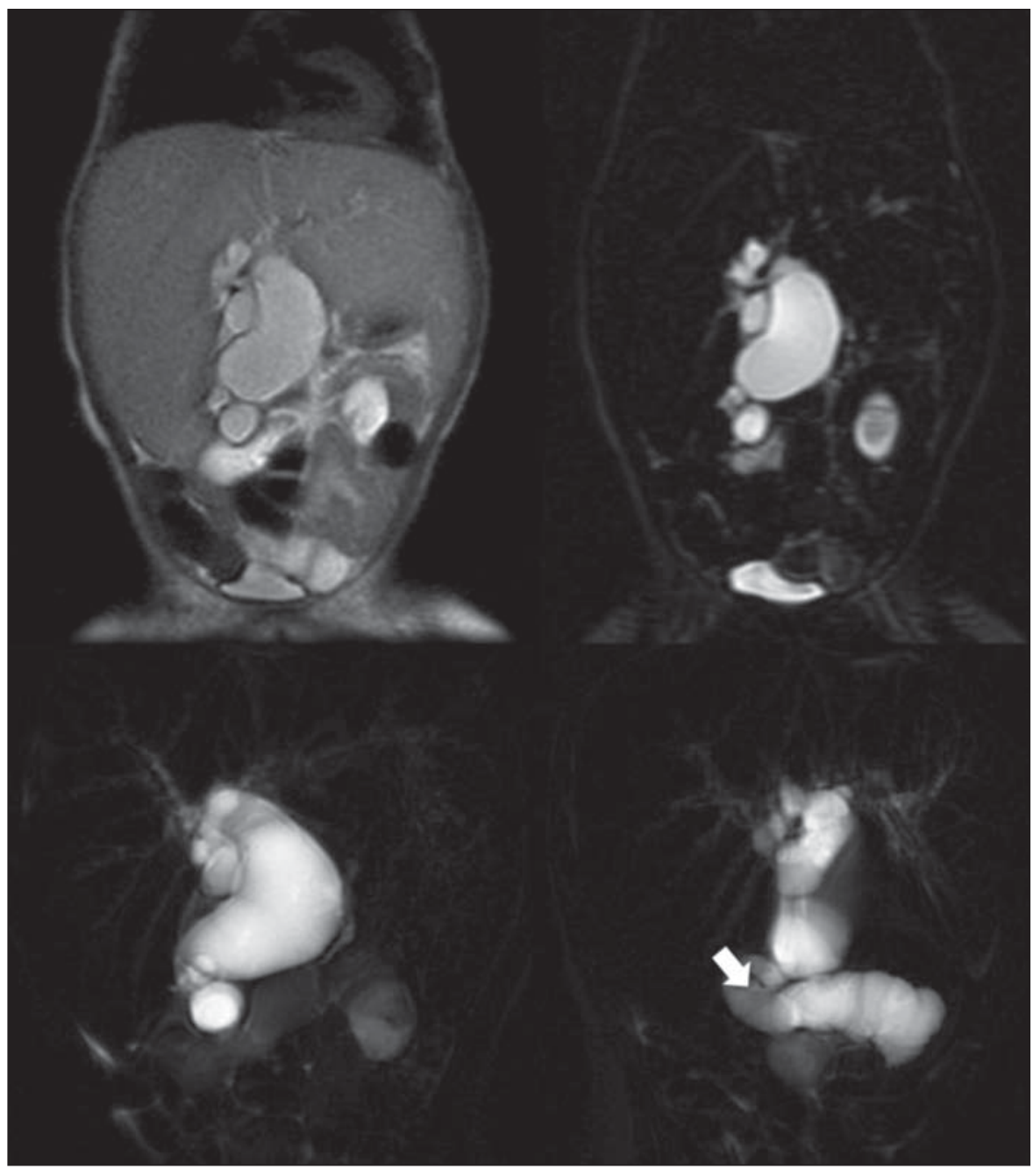




\section{Congenital Biliary Cysts}

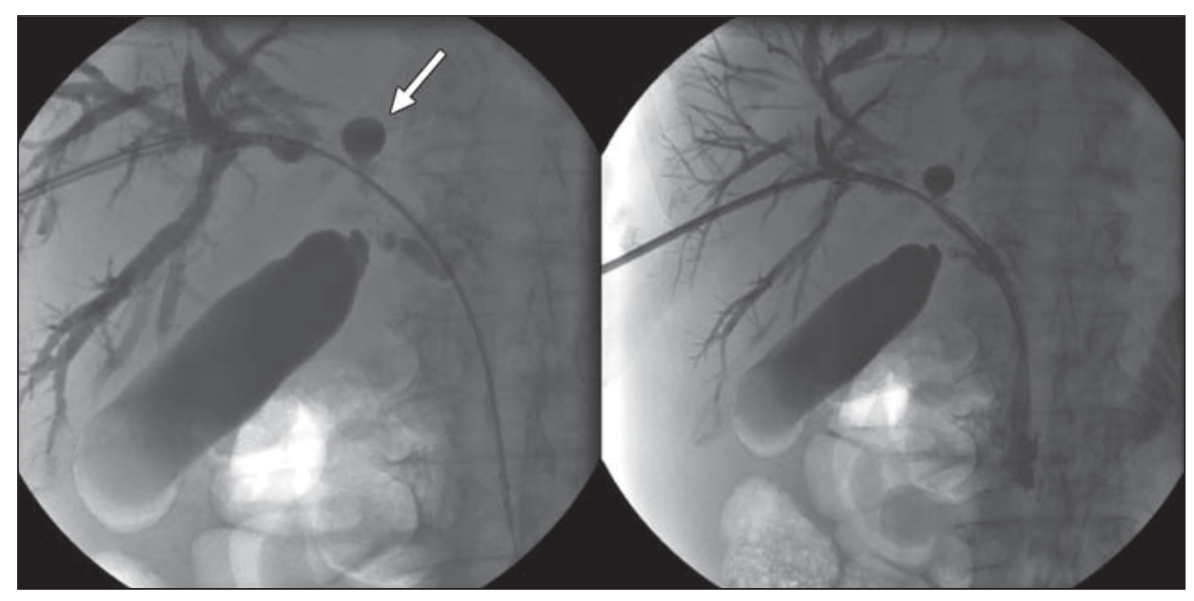

Fig. 11-Type II choledochal cyst. 76-year-old woman with known Bismuth IV Klatskin tumor. Percutaneous transhepatic cholangiogram obtained at stent placement shows small diverticulum of irregularly stenosed common hepatic duct (arrow).

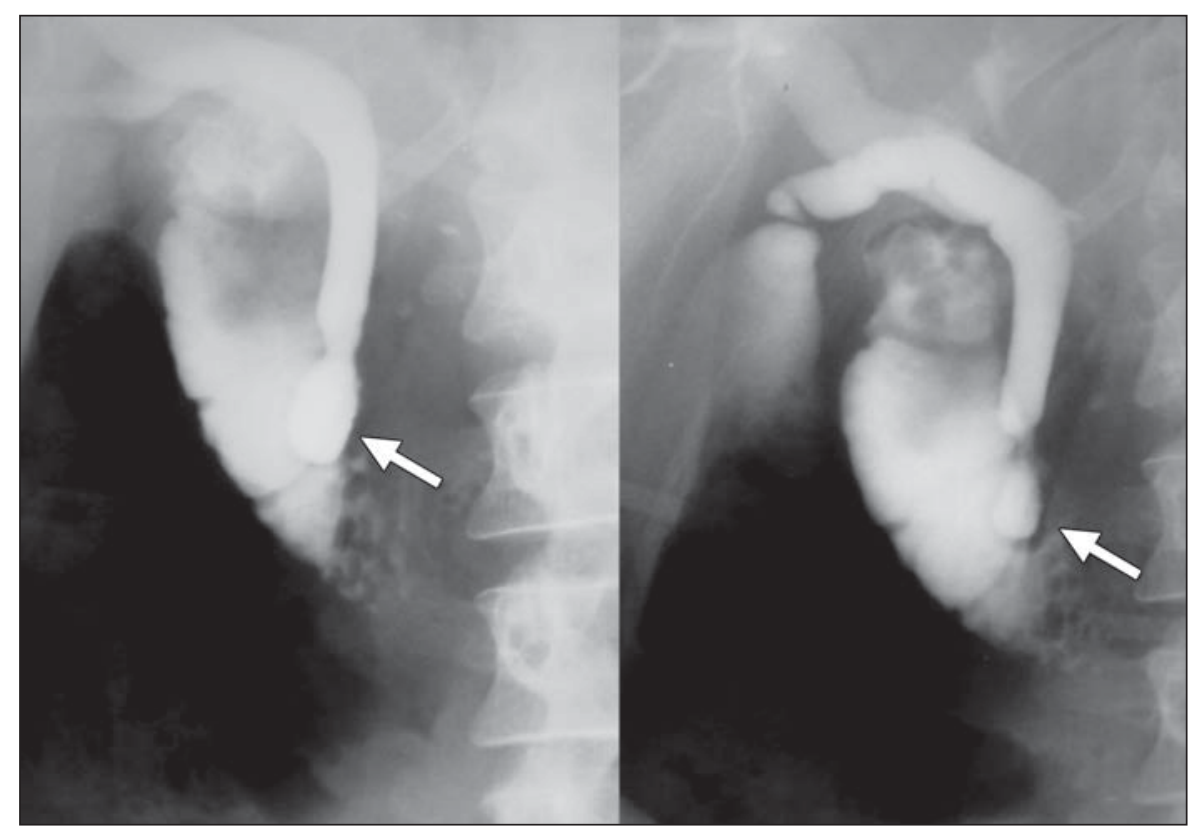

Fig. 12-Type III choledochal cyst. 42-year-old man with cholestasis. Percutaneous transhepatic cholangiogram shows diverticulum of common bile duct within wall of second portion of duodenum adjacent to papilla of Vater (arrows). 


\section{Santiago et al.}

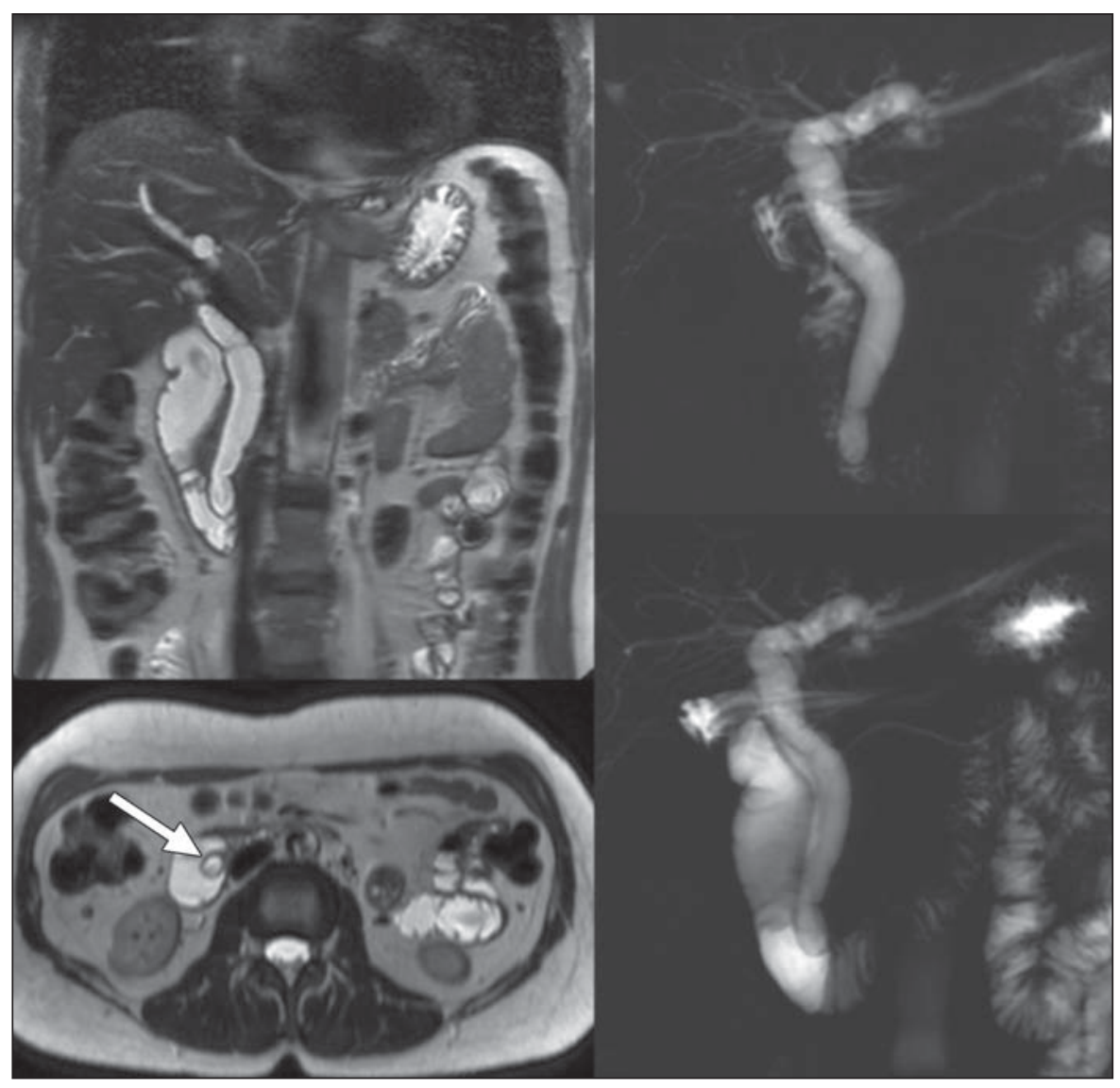

Fig. 13-Type III choledochal cyst. 55-year-old woman with right upper quadrant pain and dyspepsia. A, T2-weighted fast spin-echo (left) and thick-section single-shot fast spin-echo heavily T2-weighted (right) MR images show dilatation of intramural portion of main bile duct and protrusion of papilla into lumen of duodenum (arrow). Proximal biliary tree is moderately dilated.

B, ERCP image (left) shows regular, normal-caliber main pancreatic duct. Endoscopic photograph (right) shows draining orifice of main bile duct at apex of protruding papilla consistent with choledochocele and excluding other causes, namely intramural pseudocyst and enteric duplication cyst.
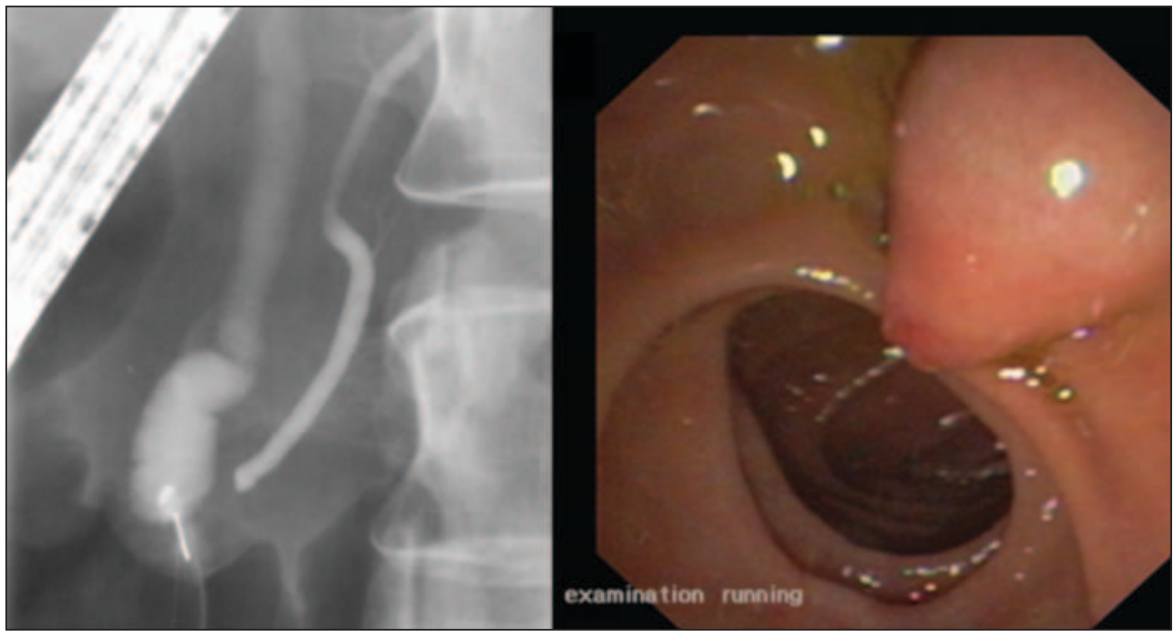


\section{Congenital Biliary Cysts}

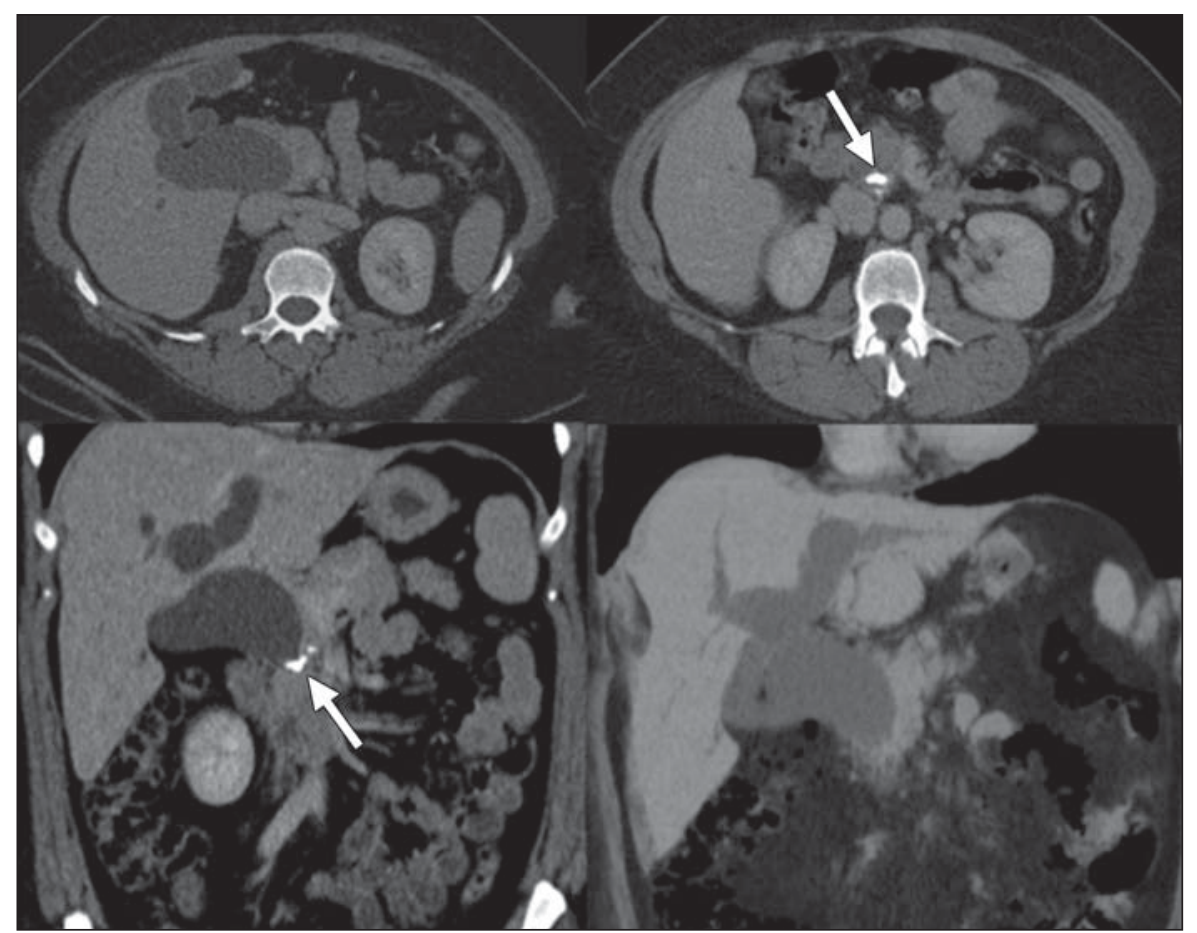

A

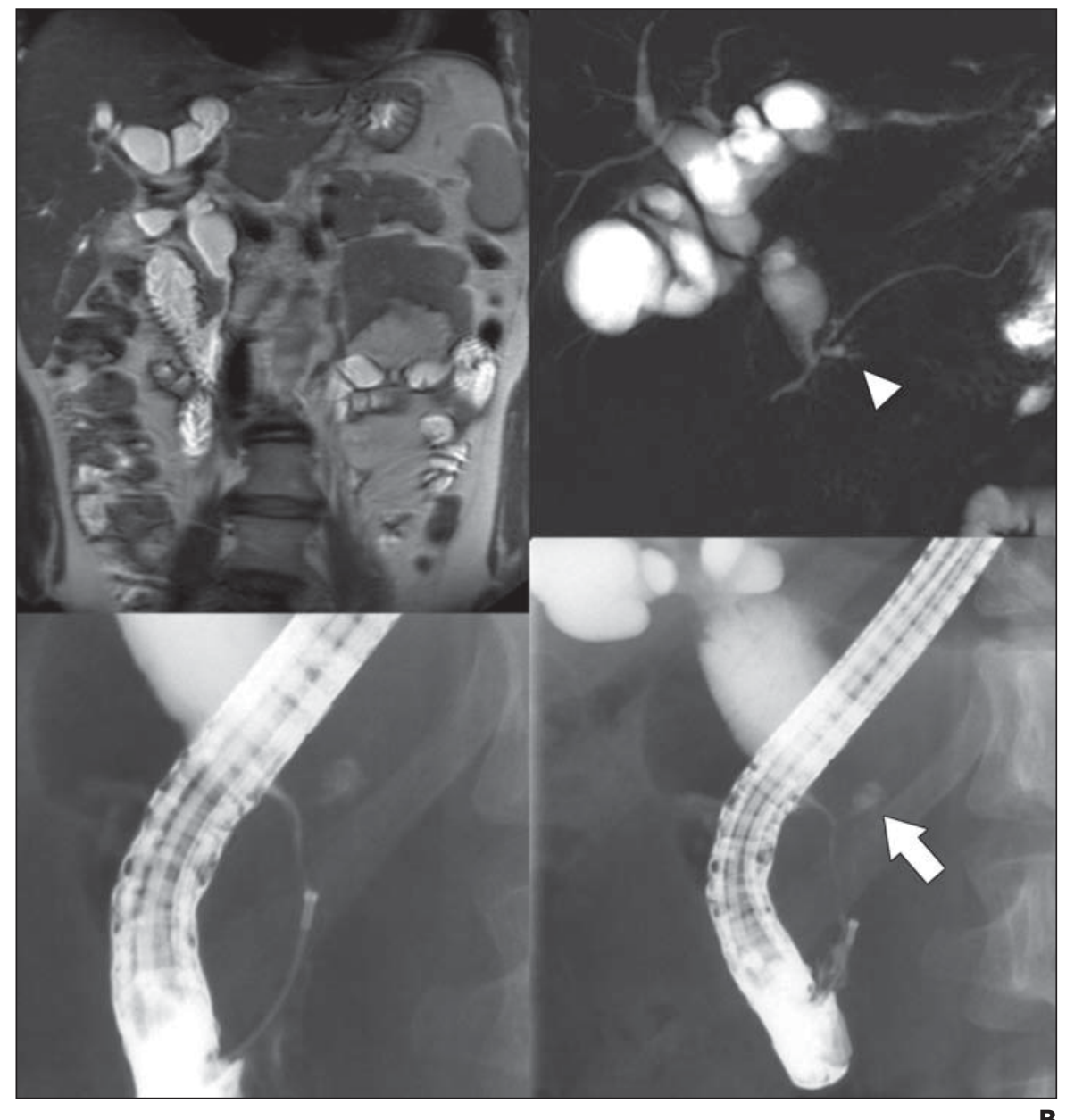

Fig. 14-Type IVA choledochal cyst. 39-year-old woman with mild and transient cholestasis detected during routine laboratory evaluation.

A, Axial (top), coronal multiplanar reformatted (bottom left), and coronal minimum-intensity projection (bottom right) reformatted MDCT images show marked fusiform dilatation of extrahepatic bile duct and irregular dilatation of proximal intrahepatic bile ducts. Irregular pancreatic calcification (arrows) is adjacent to distal ectatic portion of extrahepatic bile duct.

B, Coronal fast spin-echo T2-weighted (top left) and thick-section single-shot fast spin-echo heavily T2-weighted (top right) MR images show normal peripheral tapering of intrahepatic bile ducts favoring type IV choledochal cyst over luminal obstruction. Pancreas divisum and abnormal pancreatobiliary duct junction (long common channel) are evident, as are small number of irregularly dilated pancreatic side ducts (arrowhead) strictly in head of pancreas consistent with chronic focal pancreatitis. Main pancreatic duct is regular and of normal caliber. ERCP images (bottom) obtained after intubation of papilla of Vater show that irregular calcification corresponded to obstructive calculus in lumen of ventral pancreatic side duct (arrow) draining to long common channel. Chronic pancreatitis is thus confined to ventral portion of pancreas.

\section{FOR YOUR INFORMATION}

This article is available for CME credit. Log onto www.arrs.org; click on AJR (in the blue Publications box); click on the article name; add the article to the cart; proceed through the checkout process. 